\title{
Role of Laser Power, Wavelength, and Pulse Duration in Laser Assisted Tin-Induced Crystallization of Amorphous Silicon
}

\author{
V. B. Neimash $\left(D,{ }^{1}\right.$ A. O. Goushcha $\left(\mathbb{D},{ }^{1}\right.$ L. L. Fedorenko, ${ }^{1}$ P. Ye. Shepelyavyi $\left(\mathbb{D},{ }^{2}\right.$ \\ V. V. Strelchuk, ${ }^{2}$ A. S. Nikolenko, ${ }^{2}$ M. V. Isaiev $\left({ }^{1},{ }^{3}\right.$ and A. G. Kuzmich ${ }^{3}$ \\ ${ }^{1}$ Institute of Physics, National Academy of Sciences of Ukraine, Nauky Ave. 46, Kyiv 03028, Ukraine \\ ${ }^{2}$ V.E. Lashkaryov Institute of Semiconductor Physics, National Academy of Sciences of Ukraine, Nauky Ave. 41, Kyiv 03028, Ukraine \\ ${ }^{3}$ Faculty of Physics, Taras Shevchenko National University of Kyiv, Volodymyrska Str. 64/13, Kyiv 01601, Ukraine \\ Correspondence should be addressed to V. B. Neimash; neimash@gmail.com and A. O. Goushcha; goushcha@cox.net
}

Received 11 November 2017; Revised 7 January 2018; Accepted 22 January 2018; Published 19 February 2018

Academic Editor: Bo Tan

Copyright (c) 2018 V. B. Neimash et al. This is an open access article distributed under the Creative Commons Attribution License, which permits unrestricted use, distribution, and reproduction in any medium, provided the original work is properly cited.

\begin{abstract}
This work describes tin-induced crystallization of amorphous silicon studied with Raman spectroscopy in thin-film structures Si$\mathrm{Sn}$-Si irradiated with pulsed laser light. We have found and analyzed dependencies of the nanocrystals' size and concentration on the laser pulse intensity for $10 \mathrm{~ns}$ and $150 \mu \mathrm{m}$ duration laser pulses at the wavelengths of $535 \mathrm{~nm}$ and $1070 \mathrm{~nm}$. Efficient transformation of the amorphous silicon into a crystalline phase during the $10 \mathrm{~ns}$ time interval of the acting laser pulse in the $200 \mathrm{~nm}$ thickness films of the amorphous silicon was demonstrated. The results were analyzed theoretically by modeling the spatial and temporal distribution of temperature in the amorphous silicon sample within the laser spot location. Simulations confirmed importance of light absorption depth (irradiation wavelength) in formation and evolution of the temperature profile that affects the crystallization processes in irradiated structures.
\end{abstract}

\section{Introduction}

Layered composites utilizing nanocrystalline silicon dispersed within an amorphous Si matrix are considered to be very promising for the next generation of quantum dotsbased solar cells [1]. Such composites (referred to as nc-Si in the text below) pose a number of properties important for the photoelectric conversion of solar radiation, for example, quasi-direct bandgap mechanism of light absorption, dependence of the energy bandgap on the nanocrystals size, stability against the Stabler-Vronsky effect, and a possibility of manufacturing on flexible substrates.

Employing nc-Si as the base material allows enhancing significantly the efficiency of solar cells due to formation of polymorphic cascade heterostructures $[2,3]$ and decreasing their production cost due to advantages of the thin-film roll technology $[4,5]$. Among the hurdles that block widescale utilization of nc-Si advantages in practice is poorly developed methods of control of the nanocrystals' size and concentration at economically relevant rates of the composite film formation. That is why despite a large number of already developed methods of nc-Si fabrication, still much attention is paid and efforts are exerted to improve the existing methods and to develop new ones (see, e.g., [6-12]).

A promising path in this respect relies on the effect of metal-induced crystallization (MIC) of the amorphous $\mathrm{Si}(\alpha-\mathrm{Si})$ [13-17]. In particular, efficient formation of $\mathrm{Si}$ nanocrystals with the sizes of $2-7 \mathrm{~nm}$ and partial volume of a crystalline phase of up to $80 \%$ was demonstrated in the recent experiments on low-temperature crystallization of $\alpha$-Si with Tin (Sn) [18-20]. The experimental findings of those referenced works were modeled and explained in [20-22] by suggesting the crystallization mechanism that is essentially different from those known for Si crystallization assisted by other metals $[13,15-17]$. In accordance with the proposed mechanism, $\mathrm{Si}$ nanocrystals are formed during self-sustained cyclic (repeatable) processes of formation and decay of the supersaturated solution of silicon in tin within a thin eutectic layer at the interface of $\alpha$-Si and Sn. Further development of the proposed mechanism confirmed that 
TABLE 1: Samples' parameters and protocols of their laser treatment.

\begin{tabular}{|c|c|c|c|c|}
\hline $\begin{array}{l}\text { Sample } \\
\text { number }\end{array}$ & $\begin{array}{l}\text { Layers thickness } \\
X-Y-Z \text { in nm }\end{array}$ & $\begin{array}{l}\text { Laser light } \\
\text { wavelength }\end{array}$ & $\begin{array}{c}\text { Laser pulse } \\
\text { duration (FWHM) }\end{array}$ & $\begin{array}{c}\text { Range of laser } \\
\text { irradiation power in } \\
\mathrm{W} / \mathrm{cm}^{2}\end{array}$ \\
\hline 1 & $50-100-200$ & $1070 \mathrm{~nm}$ & $150 \mu \mathrm{s}$ & $(1.4-2.9) \times 10^{4}$ \\
\hline \multirow{2}{*}{2} & $50-100-200$ & \multirow{2}{*}{$1070 \mathrm{~nm}$} & $150 \mu \mathrm{s}$ & $1.3 \times 10^{5}$ \\
\hline & $50-100-200$ & & $10 \mathrm{~ns}$ & $(5.3-18.0) \times 10^{7}$ \\
\hline 3 & $50-100-200$ & $1070 \mathrm{~nm}$ & $150 \mu \mathrm{s}$ & $(2.9-7.8) \times 10^{4}$ \\
\hline 4 & $50-100-200$ & $535 \mathrm{~nm}$ & $10 \mathrm{~ns}$ & $(5.5-8.5) \times 10^{6}$ \\
\hline 5 & $50-100-200$ & $535 \mathrm{~nm}$ & $10 \mathrm{~ns}$ & $(8.5-21.75) \times 10^{6}$ \\
\hline 6 & $50-100-200$ & $1070 \mathrm{~nm}$ & $150 \mu \mathrm{s}$ & $(2.3-6.9) \times 10^{4}$ \\
\hline $6-1$ & $50-100-200$ & $1070 \mathrm{~nm}$ & $10 \mathrm{~ns}$ & $(7.4-15.3) \times 10^{7}$ \\
\hline $6-2$ & $50-100-200$ & $535 \mathrm{~nm}$ & $10 \mathrm{~ns}$ & $(3.5-10.3) \times 10^{6}$ \\
\hline 7 & $0-100-200$ & $1070 \mathrm{~nm}$ & $10 \mathrm{~ns}$ & $(8.4-52.0) \times 10^{7}$ \\
\hline 8 & $0-100-200$ & $1070 \mathrm{~nm}$ & $10 \mathrm{~ns}$ & $(2.0-21.8) \times 10^{7}$ \\
\hline
\end{tabular}

Sn-induced crystallization of $\alpha$-Si accelerates when laser radiation is applied to the solution [23]. In addition, applying laser radiation allowed online detection of the Raman spectra providing thereby the capabilities to monitor the temperature, partial volume, and nanocrystal sizes in nc-Si composite during its formation and showing the possibility to control efficiently the crystallization process by varying the intensity and irradiation time of laser light.

This work focuses on finding the thermal and time evolution parameters of MIC in the interfacial system $\alpha$ $\mathrm{Si} / \mathrm{Sn}$, evaluating the role of photoionization processes in Si nanocrystallites formation, and exploring possible advantages of employing a pulsed laser radiation to control the nanocrystals' size and partial volume during Sn-induced crystallization of $\alpha$-Si.

\section{Experiment}

In this work, we studied the layered structures shown schematically in Figure 1. The structures were prepared by consecutive deposition of $\mathrm{Si}$ and $\mathrm{Sn}$ through thermal evaporation in vacuum on the substrate of crystalline $\mathrm{Si}$ (c-Si) at the temperature between $150^{\circ} \mathrm{C}$ and $200^{\circ} \mathrm{C}$. The consecutive deposition of three layers ( $\mathrm{Si}, \mathrm{Sn}$, and $\mathrm{Si}$ ) was made during a single run in a vacuum chamber at $10^{-3} \mathrm{~Pa}$ without breaking the vacuum. The purity of the targets used for vacuum deposition was $99.999 \% \mathrm{Si}$ and $99.92 \% \mathrm{Sn}$. The layers' thickness for different samples and parameters of laser radiation used to assist crystallization are shown in Table 1.

In each sample, we allocated multiple regions of $0.5 \mathrm{~cm} \times$ $0.5 \mathrm{~cm}$ in size and each region of the sample was irradiated separately using the laser radiation at certain wavelength, pulse duration, and pulse power density as shown in Table 1. The laser beam (70 $\mu \mathrm{m}$ diameter laser spot) was scanned with $50 \mu \mathrm{m}$ step size in $x$ and $y$ directions. Note that each spot of the sample's surface received only one laser pulse and the entire surface of each region of the sample was exposed to identical irradiation conditions. However, different regions of the sample were exposed to different irradiation protocols

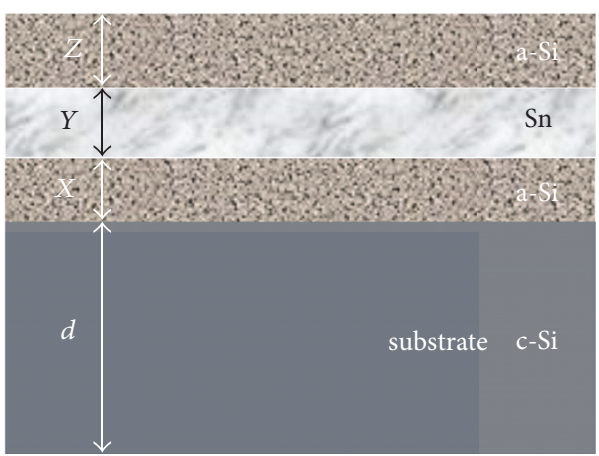

FIGURE 1: Cross-sectional schematics of Si-Sn-Si deposited samples studied in this work.

as seen from Table 1 . The applied irradiation power density ranged from $1.4 \times 10^{4}$ to $2.18 \times 10^{8} \mathrm{~W} / \mathrm{cm}^{2}$ and the pulse width was either $10 \mathrm{~ns}$ or $150 \mu \mathrm{s}$ in different runs. The laser wavelengths used were $535 \mathrm{~nm}$ and $1070 \mathrm{~nm}$. Note that samples \#3 and \#6 differed only by the range of the laser power variation. We kept these two samples separately in Table 1 to demonstrate the repeatability of the crystallization threshold in different experiments.

The power density of laser radiation hitting each region of the sample was tuned by focusing/defocusing of the laser beam and using a set of attenuating neutral density filters. As a result, each studied sample contained a number of regions irradiated with the same laser pulse duration and same wavelength but different light power density.

After irradiation, the phase composition of each region of the samples was studied by Raman spectroscopy. Raman spectra were recorded at room temperature using a backscattered geometry with the spectrometer HORIBA Jobin Yvon T64000 equipped with the confocal microscope Olympus BX41 and cooled detector. For excitation of Raman scattering, we used Ar-Kr laser at $\lambda_{\mathrm{ex}}=488 \mathrm{~nm}$. The spectral resolution of Raman spectra runs was $0.15 \mathrm{~cm}^{-1}$. The excitation light was focused on the surface of a sample 


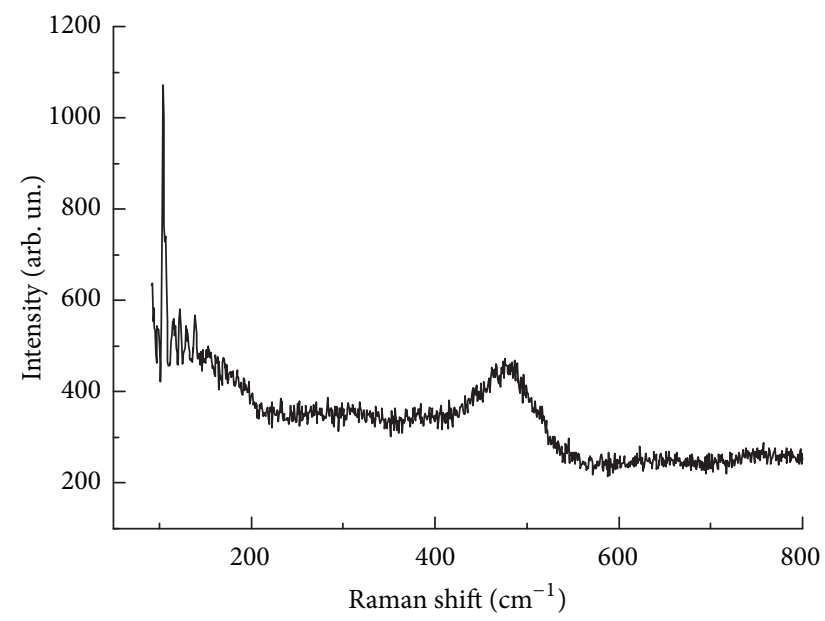

(a)

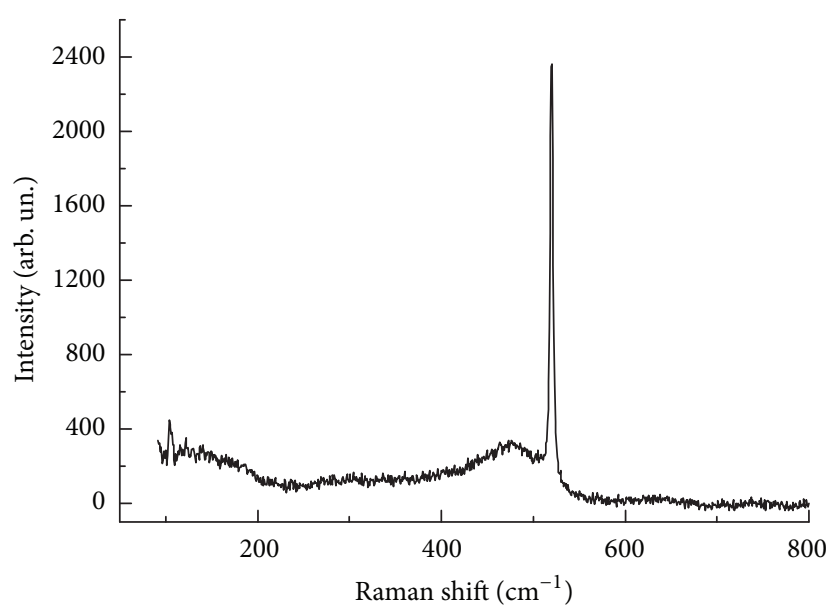

(b)

FIGURE 2: Typical Raman spectra of $\alpha$-Si (a) and partially crystallized Si (b).

in the spot size of approximately $5 \mu \mathrm{m}^{2}$ using Olympus $10 x / 0.25$ objective. The power density of Raman excitation on the surface of each sample was approximately $20 \mathrm{~kW} / \mathrm{cm}^{2}$, which did not cause any noticeable laser-induced heating beyond the room temperature. In addition, the optical images of the samples surface within the areas of Raman spectra measurements were also recorded.

\section{Results and Discussion}

Figure 2 shows typical Raman spectra within the wavenumbers range of $100-850 \mathrm{~cm}^{-1}$ of as-grown, not irradiated samples. The spectra of samples \#7 and \#8 (deposited on the substrates preheated to $150^{\circ} \mathrm{C}$ ) prior to laser irradiation treatment revealed only a broad band with a maximum centered around $475 \mathrm{~cm}^{-1}$, which is a characteristic of $\alpha$-Si [24]. Following laser treatment, the samples revealed also an additional narrow band peaked at around $500-520 \mathrm{~cm}^{-1}$, which was a signature of the nanocrystalline phase of $\mathrm{Si}$ $[24,25]$. This phase was formed as a result of MIC from the amorphous Si phase, in which the nc-Si phase formation was facilitated by laser irradiation [23]. The initial (as-grown) Raman spectra of the samples from \#1 to \#6-2 contained both the $\alpha$-Si and nc-Si Raman bands. The existence of the ncSi phase in as-deposited samples is understood when taking into account the fact that the substrates of these samples were preheated to $200^{\circ} \mathrm{C}$. During the deposition of Si atomic layers, the substrates were further heated by the evaporator radiation and the substrate temperature could exceed the Sn melting temperature $\left(232^{\circ} \mathrm{C}\right)$, leading thereby to partial crystallization of $\mathrm{Si}$. These samples were used to study possible impact of laser irradiation on the preexisted nanocrystalline phase.

The nanocrystals' size and their partial volume were evaluated from the best-fit analysis of Raman spectra simulations using the approximation of spatially confined phonons [24, 25 ] as it was described in our recent work [20]. In particular, we have found that for samples \#1, 2, 3, 6, and 6-1 the initial (prior to laser irradiation) state of the crystalline phase was characterized with the dominant size of the crystallites $L=$ $1.5 \mathrm{~nm}$ and partial volume of the crystalline phase $X_{c}=48 \%$. As is known, the largest Raman shift in comparison with the peak position for the monocrystalline $\mathrm{Si}$ is obtained for the nanocrystals' size less than $10 \mathrm{~nm}$. With the nanocrystals' size increase, this shift asymptotically approaches zero. The spectral resolution of the equipment used in our experiments was $\sim 0.15 \mathrm{~cm}^{-1}$, which allowed securely estimating the crystals' size of up to $\sim 35 \mathrm{~nm}$, which agreed with the correlation length of optical phonons in Si reported previously in some works [26]. Below we discuss in detail the evolution of these parameters upon irradiation of samples with single laser pulses of the varied intensity, duration, and wavelength.

3.1. Effect of Irradiation Power at $\lambda_{\mathrm{ex}}=1.07 \mu \mathrm{m}$ and $\tau_{p}=150$ ns. Figure 3 shows dependence of the nanocrystals size and partial volume on the irradiation power when scanning samples \#3 and \#6 with single laser pulses. Both parameters, the nanocrystals' size and their partial volume, started increasing when the irradiation power surpassed the level of $\sim 5.5 \times 10^{4} \mathrm{~W} / \mathrm{cm}^{2}$. In particular, the irradiation power increase by $42 \%$ from $5.5 \times 10^{4}$ to $7.8 \times 10^{4} \mathrm{~W} / \mathrm{cm}^{2}$ caused the nanocrystals' size increase by $230 \%$ from $1.5 \mathrm{~nm}$ to $5.0 \mathrm{~nm}$. The partial volume of the crystalline phase changes also sharply. Note larger scattering of $X_{c}$ in comparison with $L$ values caused by a larger statistical error of $X_{c}$ calculation.

These results are in a complete agreement with recently reported data on MIC effects in Si-Sn-Si structures, obtained upon irradiation with the continuous-wave laser [23]. The explicit threshold of the dependence in Figure 3 at the irradiation power density of approximately $5 \times 10^{4} \mathrm{~W} / \mathrm{cm}^{2}$ may be attributed to reaching the Sn melting point in the studied structures. In accordance with previous reports, melting of $\mathrm{Sn}$ is a prerequisite for MIC of $\alpha$-Si [22].

Upon increase of the irradiation power from $5 \times 10^{4}$ to $8 \times 10^{4} \mathrm{~W} / \mathrm{cm}^{2}$ and related increase of the sample's local temperature within the laser spot, the value of the parameter $X_{c}$ grew markedly slower $(\sim 40 \%$ total growth) than that 


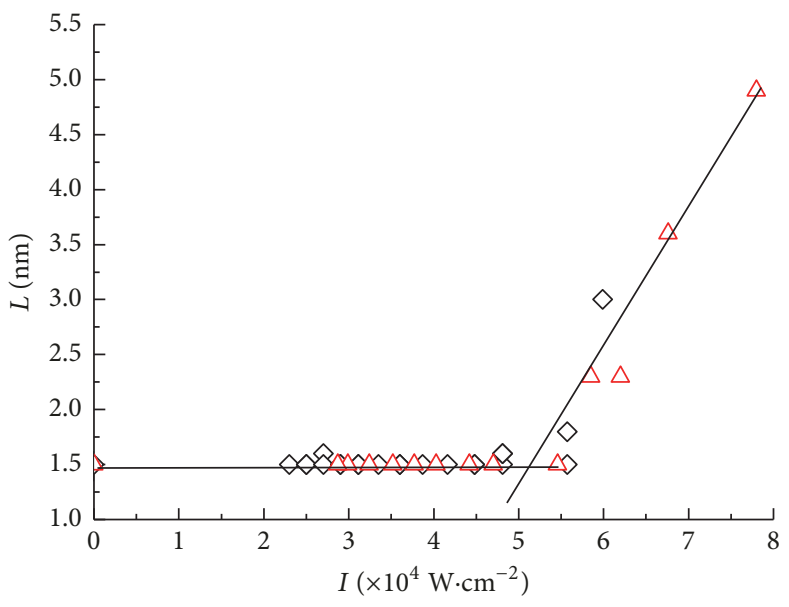

Sample: $\triangle \# 3$

$\diamond \# 6$

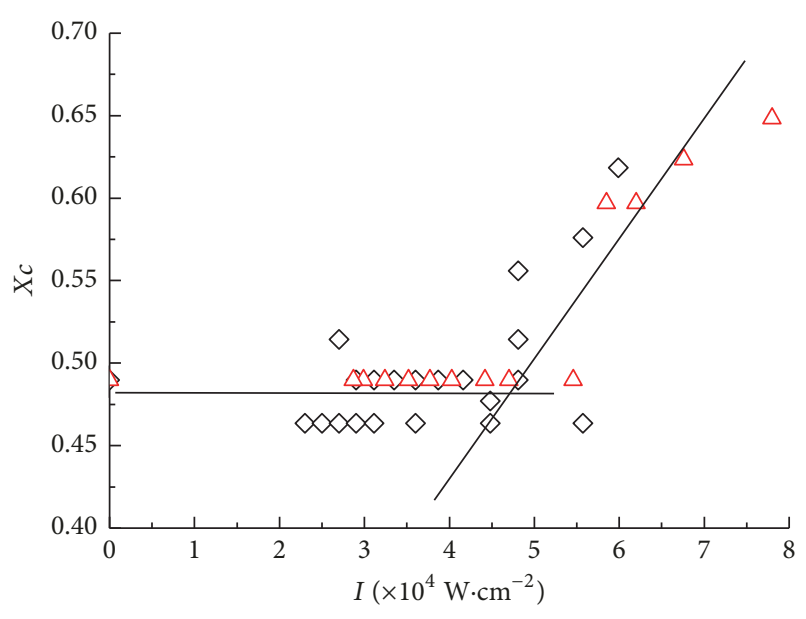

Sample: $\triangle \# 3$

$\diamond \# 6$

(a)

(b)

FIGURE 3: Dependencies of the nanocrystals size (a) and partial volume of the crystalline phase (b) on the power density of laser irradiation. Open red triangles and black diamonds show dependencies for samples \#3 and \#6, respectively.

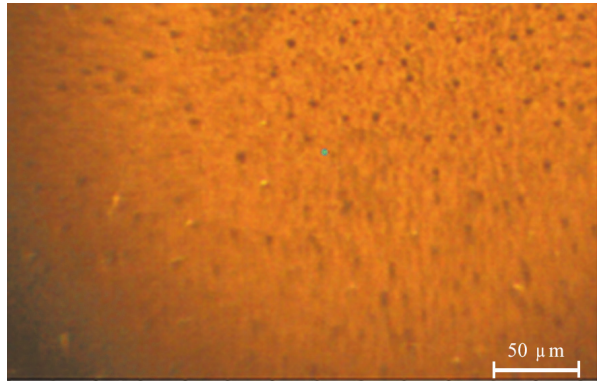

(a)

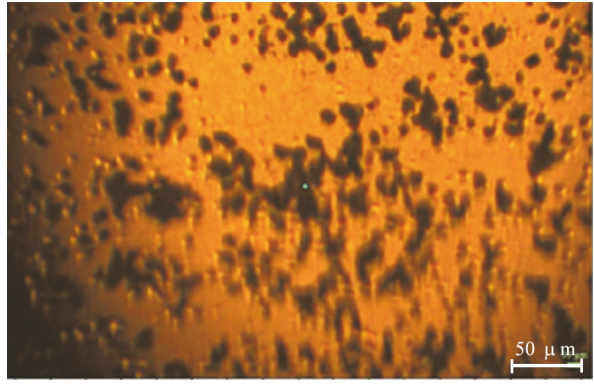

(b)

FIGURE 4: Typical optical images of the samples' surface after laser irradiation with optical power $I=0-5 \times 10^{4} \mathrm{~W} / \mathrm{cm}^{-2}(\mathrm{a})$ and $I=8.2 \times$ $10^{4} \mathrm{~W} / \mathrm{cm}^{-2}$ (b). Irradiation wavelength was $\lambda_{\mathrm{ex}}=1.07 \mu \mathrm{m}$ and pulse duration $\tau_{p}=150 \mathrm{~ns}$.

of the parameter $L$ ( $230 \%$ total growth). This was rather unexpected, since the volume of a crystal grows as $\sim L^{3}$. We suggest that only a small portion of initially created nanocrystals participated in precipitation of Si in Sn. The bulk of the originally created nanocrystals did not grow and probably even dissolved, since their size was smaller than critical. Such behavior is typical for the decay of saturated solutions and is in line with the previously suggested crystallization mechanism [20-22].

Figure 4 shows optical images of the as-grown samples and samples irradiated using the subthreshold laser intensities (a) as well as the regions irradiated with the maximum power laser pulses (b). Because the laser spot diameter in Raman experiments was very small (close to $2 \mu \mathrm{m}$ ), we were able to analyze separately the locations of the samples revealed with the dark spots on the images and it was determined that these dark spots have higher concentration of nanocrystals in the amorphous Si matrix than that in the "light" areas of the sample.
Such dark regions with the increased concentration of nanocrystals were typical for the as-grown samples. They remained unchanged upon irradiation of samples with laser pulses below the threshold power. When the laser power exceeded the threshold, the dark regions with a higher concentration of nanocrystals became larger. An increase of the total area of the dark regions correlated with the increase in the partial volume $X_{c}$ of the nanocrystalline phase measured from the Raman spectra.

3.2. Effect of Irradiation Power at $\lambda_{\mathrm{ex}}=1.07 \mu \mathrm{m}$ and $\tau_{p}=10 \mathrm{~ns}$. Similar to the experiments described above in Section 3.1, samples \#2 and \#6-1 (with the 3-layer structure Si-Sn-Si, 50-100-200 nm) were irradiated with light pulses at $1070 \mathrm{~nm}$ but the pulse duration was four orders of magnitude shorter $(10 \mathrm{~ns})$ and the peak power was three orders of magnitude higher $\left(\sim 10^{7} \mathrm{~W} / \mathrm{cm}^{2}\right)$ than in the case described in the previous section. Figure 5 summarizes effects of such irradiation on the nc-Si parameters. The crystallization threshold 


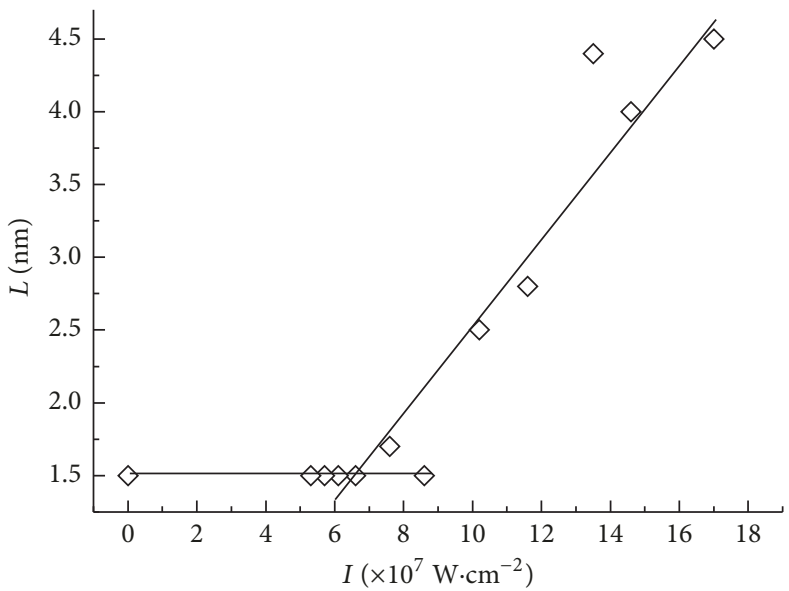

Sample: $\diamond \# 2$

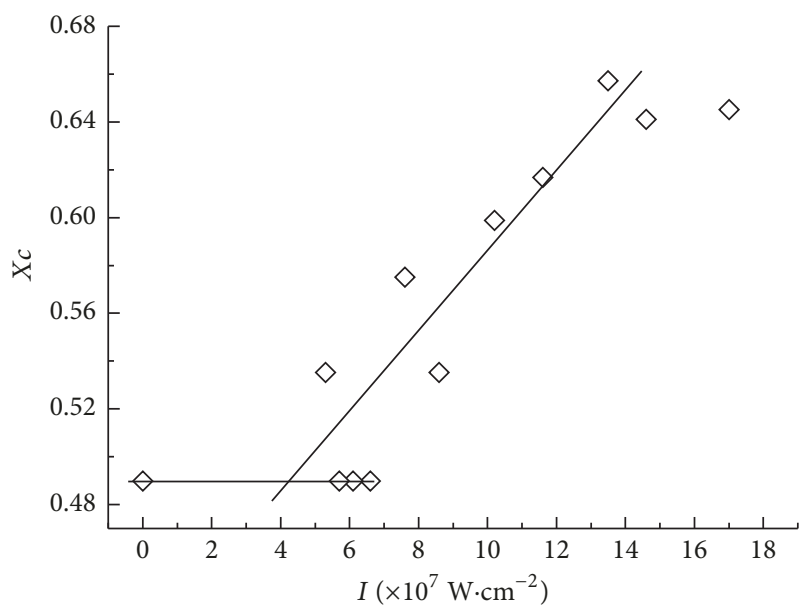

Sample: $\diamond \# 2$

(a)

(b)

Figure 5: Dependencies of the nanocrystals' size (a) and partial volume of the crystalline phase (b) on the power density of laser irradiation for sample \#2. Open black diamonds represent experimental data; solid lines show linear approximation of the data.

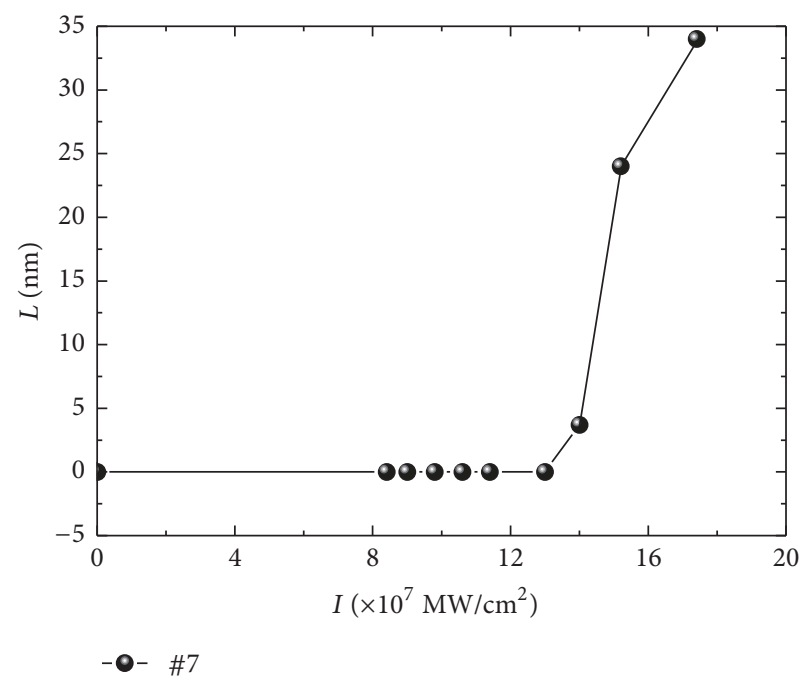

(a)

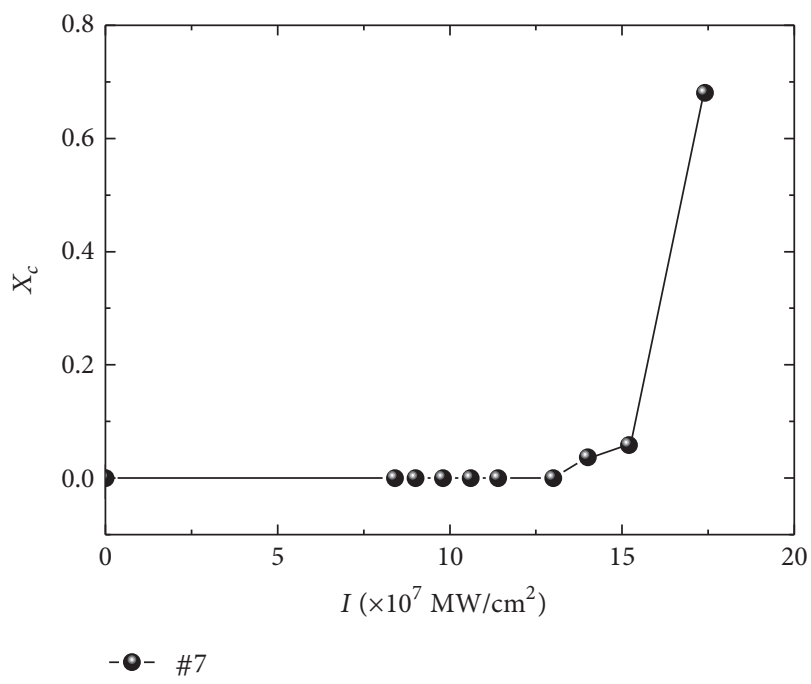

(b)

FIGURE 6: Dependencies of the nanocrystals' size (a) and partial volume of the crystalline phase (b) on the power density of laser irradiation with $10 \mathrm{~ns}$ pulse duration for sample \#7.

was observed at irradiation power of $\sim 7.5 \times 10^{7} \mathrm{~W} / \mathrm{cm}^{2}$ and the total area of crystallized regions (dark spots observed in the microscope) correlated with the laser irradiation power. The absorbed energy of optical radiation at the crystallization threshold, $E^{\text {tc }}$, can be estimated as proportional to the product of the threshold laser power density $I$ and pulse duration $\tau_{p}: E^{\text {tc }}=I \tau_{p}$. This energy was estimated as $E_{1}{ }^{\text {tc }}=$ $8.3 \mathrm{~J} / \mathrm{cm}^{2}$ for the case described in Section 3.1 and as $E_{1 I}{ }^{\text {tc }}$ $=0.75 \mathrm{~J} / \mathrm{cm}^{2}$ for the case of Section 3.2, confirming thereby that significantly lower optical energy was required to initiate MIC in the case of a higher irradiation power density. This result can be explained either by an enhanced crystallization due to photoionization effects $[23,27]$ or by more efficient heating of $\mathrm{Sn}: \mathrm{Si}$ mixture with the nanosecond compared to microsecond duration pulses caused by a limited rate of thermal dissipation $[28,29]$.

Figure 6 shows experimentally obtained dependencies of Si nanocrystals size and partial volume of nc-Si phase on the power density of $10 \mathrm{~ns}$ duration laser pulses in sample \#7. The initial state of this sample was fully amorphous. The optical irradiation with the power density below $\sim 1.5$ $\times 10^{8} \mathrm{~W} / \mathrm{cm}^{2}$ did not initiate phase transformation in the amorphous film. The nanocrystalline phase started to appear at higher irradiation intensities and the first observed crystals had sizes at the excess of $10 \mathrm{~nm}$. At optical power densities above $2.0 \times 10^{8} \mathrm{~W} / \mathrm{cm}^{2}$, the only phase detected in Raman 


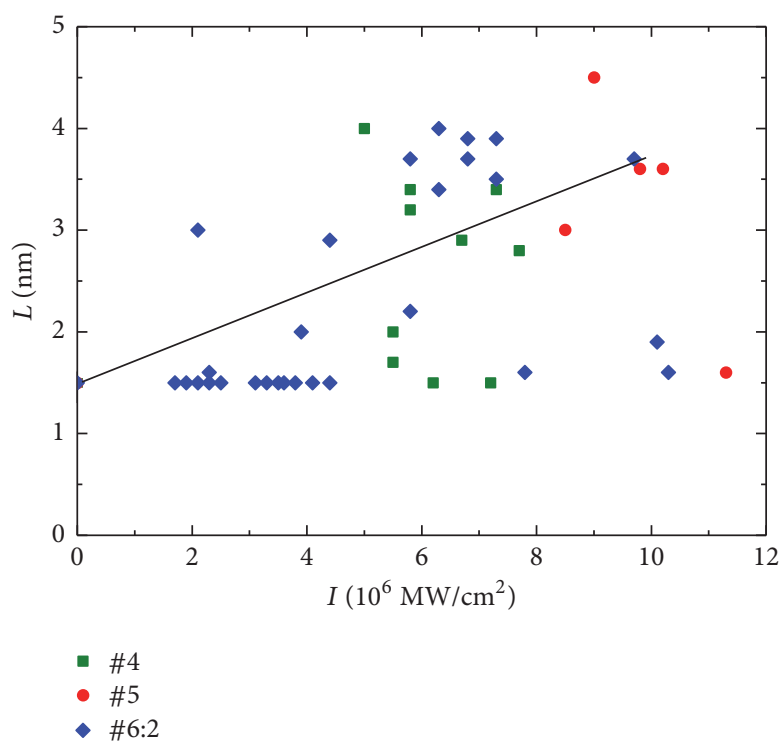

(a)

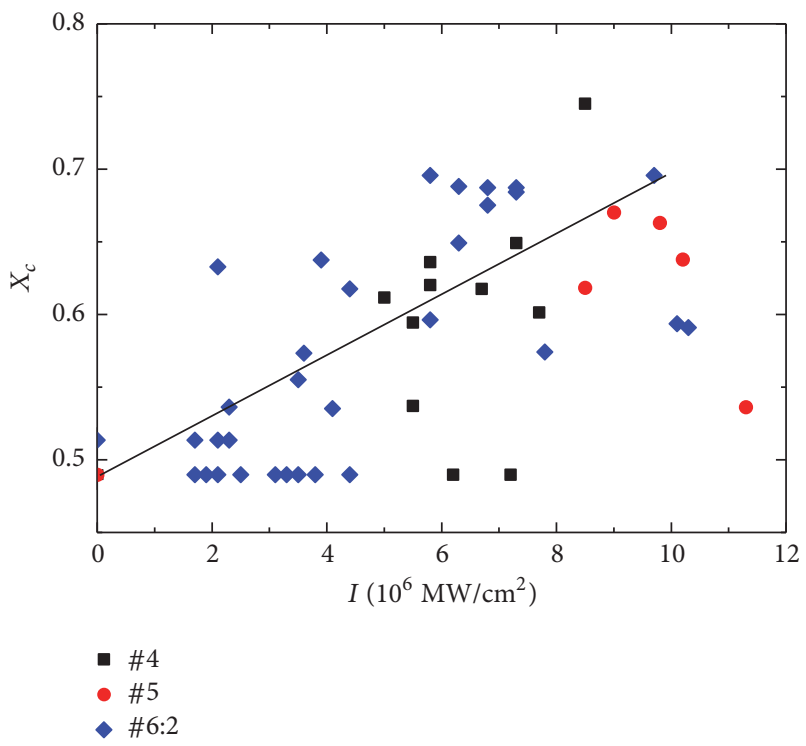

(b)

Figure 7: Dependencies of the nanocrystals' size (a) and partial volume of the crystalline phase (b) on the power density of laser irradiation with $10 \mathrm{~ns}$ pulse duration at $535 \mathrm{~nm}$. Black and green squares, red circles, and blue diamonds show data for samples \#4, \#5, and \#6.2, respectively. The trend behavior is shown with solid lines using a linear approximation.

spectra was the monocrystalline phase of the substrate (with the wave number $520 \mathrm{~cm}^{-1}$ ). Note that experimental setup allowed distinguishing securely nanocrystals with the size of up to $\sim 35 \mathrm{~nm}$. All larger size crystals were not readily distinguished from the monocrystalline $\mathrm{Si}$ in Raman spectra. These experiments with sample \#7 evidenced that the amorphous film degraded essentially without crystallization at irradiation intensities $I=(1.5-2.0) \times 10^{8} \mathrm{~W} / \mathrm{cm}^{2}$. Note that sample \#8 that also did not contain initial crystalline phase showed similar behavior.

To sum up, in contrast to samples \#2 and \#6 that already contained the nc-Si phase before irradiation and exhibited a pronounced growth of the crystalline phase partial volume with the increased irradiation intensity, the fully amorphous samples \#7 and \#8 did not show crystallization at the same irradiation conditions. This allows concluding that the MIC process goes through the incubation stage of nucleation and fast nanocrystals' growth to the sizes of $1.5-4.5 \mathrm{~nm}$ which lasts over the $10 \mathrm{~ns}$ time interval.

3.3. Effect of Irradiation Power at $\lambda_{\mathrm{ex}}=535 \mathrm{~nm}$ and $\tau_{p}=$ $10 \mathrm{~ns}$. In contrast to the $1070 \mathrm{~nm}$ wavelength light that is weakly absorbed in $\alpha$-Si and heats up primarily the Sn layer in the studied structures, the radiation at $535 \mathrm{~nm}$ is absorbed almost totally within the top layer of $\alpha$-Si in the studied structures. Samples \#4, \#5, and \#6-2 were irradiated with $10 \mathrm{~ns}$ laser pulses at $535 \mathrm{~nm}$ and with peak power density within the range of $10^{6}-10^{7} \mathrm{~W} / \mathrm{cm}^{-2}$. The results of Raman spectra analysis are shown in Figure 7.

The distribution of both the dominant crystal size $L$ and the nc-Si partial volume $X_{c}$ was significantly broader for these samples than for the samples studied in the previous sections. Such broader parameters distribution may be due to a nonuniform transformation of the outer layer of $\alpha$-Si into the nc-Si phase. Note that each point of the graphs in Figure 7 corresponds to a specific location on the sample's surface subjected to a pulsed illumination with the laser beam of $70 \mathrm{um}$ spot diameter and applying $50 \mathrm{um}$ scanning step. At the same time, the spot diameter for Raman excitation was $\sim 2$ um. Obviously, upon irradiation of a sample with $1070 \mathrm{~nm}$ light pulses, the light was absorbed primarily in the highly thermally conductive Sn layer of the structure that facilitates fast equilibration of the absorbed energy across the excitation spot. In contrast, the thermal equilibration was not achieved with the same fast rate when $535 \mathrm{~nm}$ laser pulses were used, since in this case the light absorption occurred primarily in the top $\alpha$-Si layer having lower thermal conductivity properties. In fact, the thermal diffusion length is defined as $[30,31]$

$$
l_{\mathrm{th}}=\left[(\varkappa+D) \tau_{p}\right]^{1 / 2}
$$

where $\varkappa$ is the thermal conductivity, $D$ is the ambipolar carrier diffusion in silicon, and is $\tau_{p}$ the laser pulse duration.

For Sn, $\varkappa=0.37 \mathrm{~cm}^{2} / \mathrm{sec}$ [32] and the laser pulse duration $\tau_{p}=10^{-8} \mathrm{~s} ;(1)$ results in $l_{\text {th }}=6.1 \times 10^{-5} \mathrm{~cm}$. The absorption coefficient for $535 \mathrm{~nm}$ light in $\alpha$-Si is $\alpha \approx 6.0 \times 10^{4} \mathrm{~cm}^{-1}$, resulting in the absorption length of $l_{\alpha}=1 / \alpha=1.6 \times 10^{-5} \mathrm{~cm}^{-1}$ $[33,34]$, whereas the thermal diffusion depth for $\alpha$-Si is $l_{\text {th }} \approx$ $8.0 \times 10^{-6} \mathrm{~cm}[35,36]$. The depth of heating in the direction perpendicular to the layers stack is determined by the larger of the two values $\left(l_{\alpha}\right.$ and $\left.l_{\mathrm{th}}\right)$; that is, it is determined by the absorption depth $l_{\alpha}=1.6 \times 10^{-5} \mathrm{~cm}^{-1}$. In the lateral direction, the thermal equilibration is determined by the thermal diffusion depth $l_{\text {th }} \approx 8.0 \times 10^{-6} \mathrm{~cm}$. The extent of a lateral propagation of heat in the studied samples was approximately 


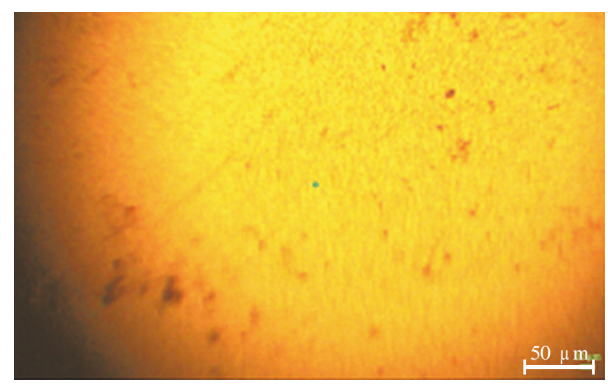

(a)

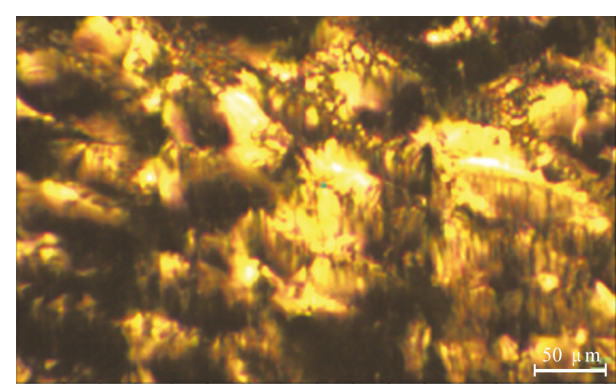

(b)

FIGURE 8: Optical images of a sample surface without laser irradiation (a) and irradiated with laser at $535 \mathrm{~nm}, 10 \mathrm{~ns}$ pulse duration, and optical power of $I=2-20 \times 10^{6} \mathrm{~W} / \mathrm{cm}^{-2}(\mathrm{~b})$.

an order of magnitude smaller than the thickness of the top layer of $\alpha$-Si. A lower thermal conductivity in these samples facilitated an increase in the maximum achieved local temperature and consequently produced more pronounced temperature gradient. In turn, this might cause higher levels of a local deformation and surface degradation of $\alpha$-Si layer in the sample. Such effects may be more pronounced at $535 \mathrm{~nm}$ irradiation with $10 \mathrm{~ns}$ duration laser pulses even though the irradiation intensity at $535 \mathrm{~nm}$ was an order of magnitude lower than that at $1070 \mathrm{~nm}$. As it is seen in the optical images of Figure 8, the laser irradiation at $535 \mathrm{~nm}$ produced rather severe changes to the surface of the irradiated samples.

\section{Simulation Results}

Let us consider a one-dimensional case of thermal equilibration along the depth of the studied structures. Use a one-dimensional thermal conductivity equation based on Fourier's law:

$$
c \rho \frac{\partial \theta}{\partial t}=\left(\frac{\partial}{\partial z} \varkappa \frac{\partial \theta}{\partial z}\right)+P(z, t),
$$

where $c$ and $\rho$ are the specific heat and density of the medium, $\varkappa$ is the thermal conductivity coefficient, and $P(z, t)$ is the function that describes the spatial and temporal distribution of extended radiation sources. Equation (2) allows for rather accurate estimation of the medium temperature within the laser spot in the case when the spot diameter exceeds significantly the thermal diffusion length [37, 38].

Using the first-order approximation, we can separate the spatial and temporal variables:

$$
P(z, t)=f(z) g(t) .
$$

The spatial distribution of the thermal sources may be written in our case as follows:

$$
f(z)=I_{0} \alpha(z) \exp \left(-\int_{0}^{z} \alpha\left(z^{\prime}\right) d z^{\prime}\right),
$$

where $\alpha(z)$ is the spatial distribution of the optical absorption coefficient, $z$ is the direction inside the depth of a sample perpendicular to its surface, and $I_{0}$ is the normalized amplitude. In all cases below, we will assume the absence of the temperature $\theta$ increase in the structure prior to optical excitation as the initial condition $(\theta(z, 0)=0)$.

Let us analyze thermal heating caused by the laser pulse of $150 \mu$ s duration. The rising and falling edges of the laser pulse are considered to be much shorter than the total length of the pulse. Hence, we can consider a $\pi$-shaped optical pulse:

$$
g(t)= \begin{cases}1, & t \leq \tau_{p} \\ 0, & t>\tau_{p},\end{cases}
$$

where $\tau_{p}=150 \mu$ s is pulse duration.

Since the pulse duration of $150 \mu$ s is much longer than the characteristic time of heat dissipation in the deposited layer of a sample, we approximate the process as the heat dissipation in the crystalline substrate. The volume sources of heat in the modified (by the optical pulse action) layer are considered as the top-surface layer sources:

$$
\left.\varkappa \frac{\partial \theta}{\partial z}\right|_{z=0}=P_{s}(t),
$$

where

$$
P_{s}(t)=\int_{0}^{X+Y+X} P(z, t) d z .
$$

In addition, we use the boundary condition of absence of the heat flow through the bottom surface of the sample:

$$
\left.\varkappa \frac{\partial \theta}{\partial z}\right|_{z=X+Y+X+D}=0 .
$$

The results of the time dependence simulations of the sample's surface temperature in accordance with the above equations and for the power density of optical irradiation of $10^{4} \mathrm{~W} / \mathrm{cm}^{2}$ are presented in Figure 9(a). Correspondingly, typical dependence of the temperature spatial distribution at $t=\tau_{p}$ is presented in Figure 9(b).

In accord with the data of Figure 5, the threshold for structural changes upon irradiation occurred close to the optical power densities of $5 \times 10^{4} \mathrm{~W} / \mathrm{cm}^{2}$, which is approximately 5 times higher than that used in calculations of Figure 9. To estimate the sample's temperature reached under 


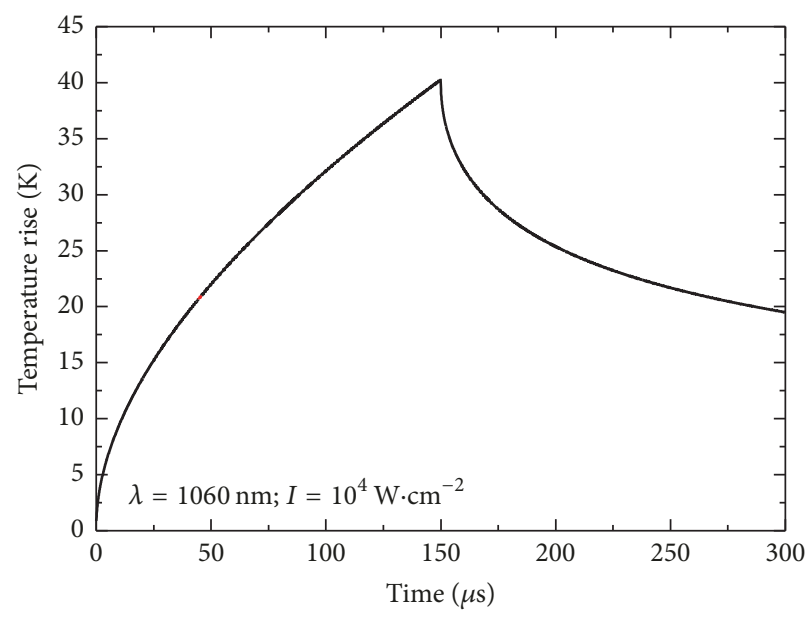

(a)

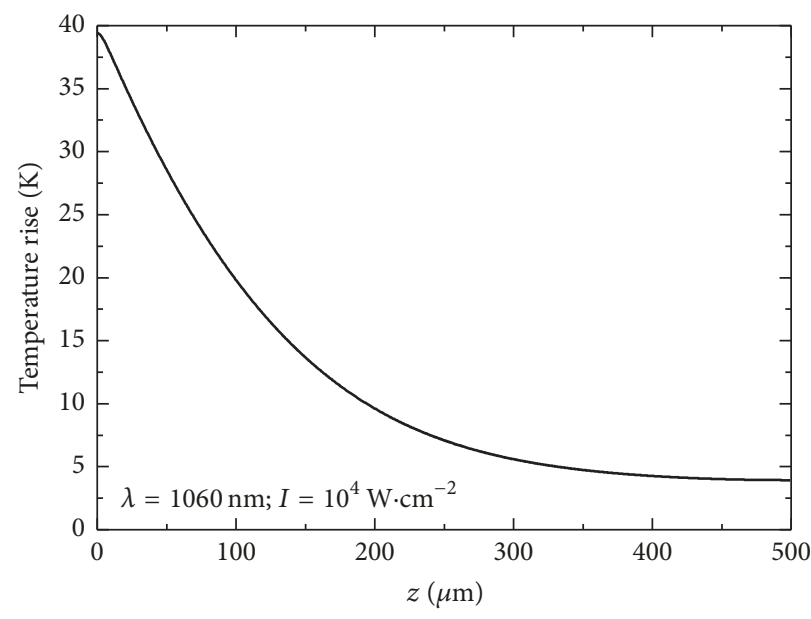

(b)

FIGURE 9: (a) Calculated surface temperature rise for irradiated samples during the laser pulse action (first $150 \mu$ s of the time scale in $x$-axis) and immediately after cessation of the irradiation. (b) Distribution of the temperature rise over the depth of a sample immediately after cessation of the laser irradiation.

irradiation intensity of $5 \times 10^{4} \mathrm{~W} / \mathrm{cm}^{2}$, we used the firstorder (linear) approximation by multiplying the temperature calculated in Figure 9 by the factor of 5 and adding the ambient temperature $(300 \mathrm{~K})$. Such calculations yielded the value of $300 \mathrm{~K}+40 \mathrm{~K} \times 5=500 \mathrm{~K}$, which is very close to the melting point of $\mathrm{Sn}(505 \mathrm{~K})$. Using the maximum optical power density of $8 \times 10^{4} \mathrm{~W} / \mathrm{cm}^{2}$ (see Figure 4 ), the calculated temperature was $300 \mathrm{~K}+40 \mathrm{~K} \times 8=620 \mathrm{~K}$, which agrees well with the previously reported data on Sn-induced crystallization of $\alpha$-Si [18-21].

When irradiating the structures with optical pulses of nanosecond duration, the specifics of spatial distribution of thermal and optical parameters of the irradiated structure play a critical role in defining the final temperature. Therefore, to get a better insight into these effects, we consider spatial distributions in more detail. In particular, Figure 10 shows the calculated spatial distribution of the thermal sources $f(z)$ and temporal distribution of temperature on the top surface of studied structures for two wavelengths $(532 \mathrm{~nm}$ and $1064 \mathrm{~nm})$ at irradiation power density of $1 \mathrm{MW} / \mathrm{cm}^{2}$.

As is seen from Figure 10(a), irradiation with $1064 \mathrm{~nm}$ laser light produces local heating related primarily to optical absorption by the layer of Sn. The temporal evolution of intensity in the laser pulse was assumed to be Gaussian:

$$
g(t)=\exp \left(-4 \log (2) \frac{\left(t-\tau_{p}\right)^{2}}{\tau_{p}^{2}}\right) .
$$

Figure 10(b) shows that, at the optical power used in the calculations $\left(1 \mathrm{MW} / \mathrm{cm}^{2}\right)$ and at $\lambda=532 \mathrm{~nm}$, the local temperature on the top surface of the illuminated structure reaches the melting point of Sn. This is in a good correlation with the results of Figure 7 -the crystallization starts at minimal intensity of optical irradiation. The optical power density of $1 \mathrm{MW} / \mathrm{cm}^{2}$ is not high enough to trigger crystallization when irradiating the sample with $\lambda=532 \mathrm{~nm}$ laser pulses. However, increasing the optical power density to $60 \mathrm{MW} / \mathrm{cm}^{2}$ (which corresponds to the experimentally determined crystallization threshold at $532 \mathrm{~nm}$, see Figure 5), the calculated temperature of the top surface layer reaches the temperature of $300 \mathrm{~K}+$ $(30 \mathrm{~K} \times 60)=2100 \mathrm{~K}$ (using the first-order (linear) approximation), which exceeds significantly the Sn melting point. Such deviation of the simulation results from the experimental data may be explained by the influence of nonlinear optical effects, in particular by the absorption saturation in studied samples [39]. One can assume that the density of electronic states decreases significantly in nanofragments compared to the bulk material, which may cause a considerable decrease in the value of the optical absorption coefficient for the nanofragments and correspondingly cause significantly lower heating. Nanostructuring of thin layers of $\mathrm{Sn}$ in the structures $\mathrm{Si}-\mathrm{Sn}$-Si was reported recently [20].

\section{Conclusions}

The fact that the starting temperature for structural changes in the studied samples of Si-Sn-Sn was very close to the melting point of Sn supports our recent conclusion on the physical mechanisms of Sn-induced crystallization in $\alpha-\mathrm{Si}$ as the cyclic process of formation and decay of a saturated solution of Si in molten Sn [20, 21]. Two stages of MIC process are suggested in this work: first the nucleation occurs during the incubation period and then fast growth of crystallites is characteristic for the processes involving the solution decay, which additionally supports the suggested mechanism.

Our results evidenced also the possibility of photoionization effects influencing the MIC process. Since the rates of Sninduced crystallization of $\alpha$-Si are rather high $\left(10^{-8}-10^{-4} \mathrm{~s}\right)$, its stimulation with the pulsed laser light may provide an insight into the method to control the nanocrystals' size during nc-Si films formation. This may find applications 


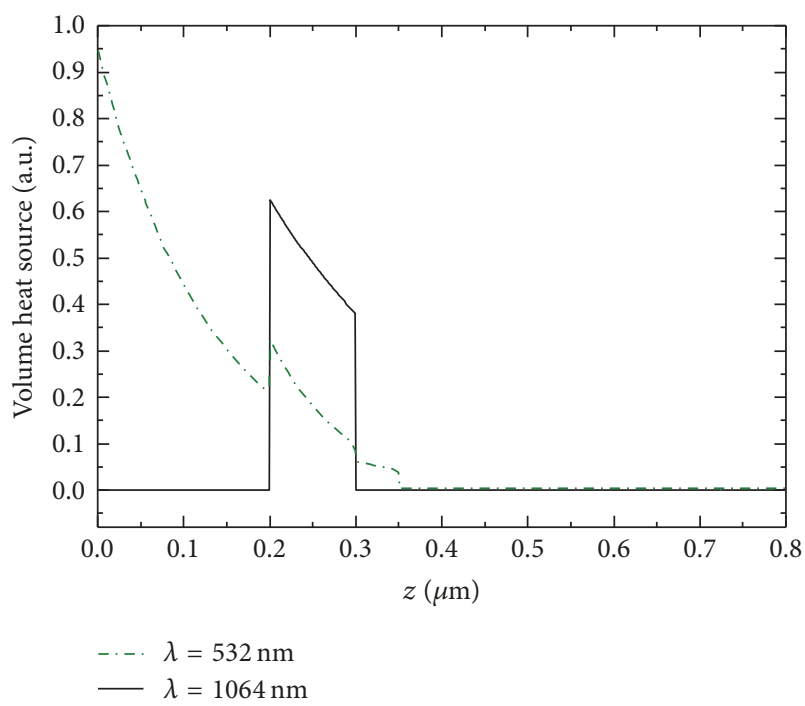

(a)

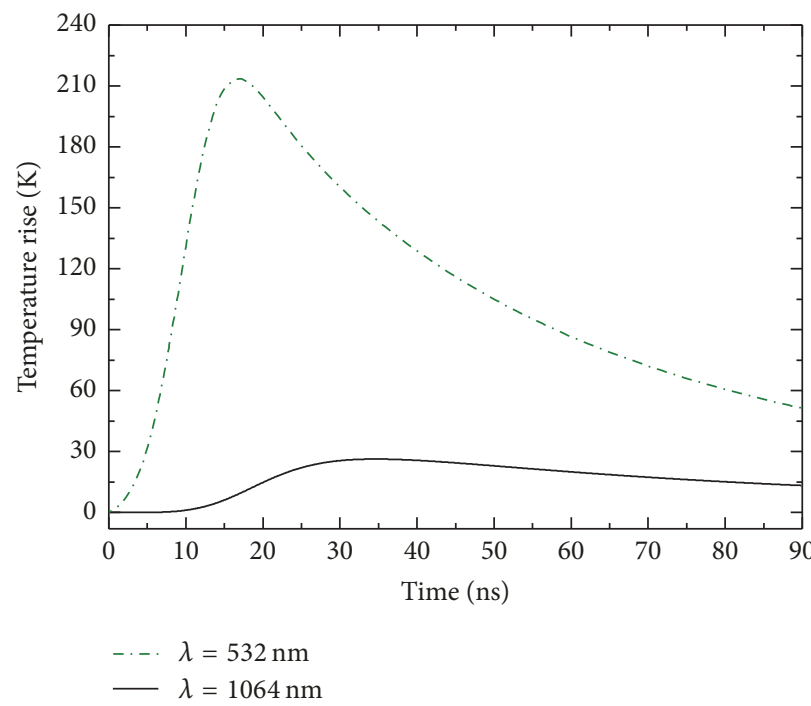

(b)

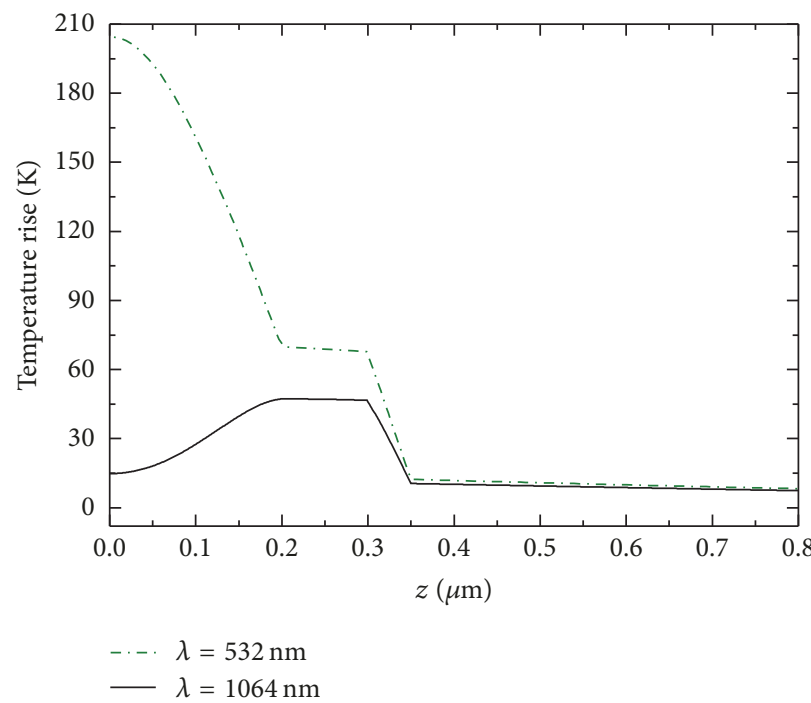

(c)

Figure 10: (a) Calculated distribution of thermal sources $f(z)$ over the depth of the structure Si-Sn-Si. (b) Temperature changes of the sample surface with time. (c) Temperature distribution at the surface of a sample at $t=2 \tau_{p}$. Irradiation with $10 \mathrm{~ns}$ duration; $1 \mathrm{MW} / \mathrm{cm}^{2}$ laser pulses at $1064 \mathrm{~nm}$ (black solid lines) and $532 \mathrm{~nm}$ (dashed green line).

not only in already mentioned technology of optical sensors and photovoltaic elements of the cascade type but also in designing of the so-called "all-Si" solar cells, in which $\mathrm{Si}$ nanoclusters are formed between the dielectric layers such as $\mathrm{SiO}_{2}, \mathrm{Si}_{3} \mathrm{~N}_{4}$, and $\mathrm{SiC}[40,41]$. Among the useful applications, we can also mention development of efficient silicon gas/fumes sensors that use nanocluster catalyzers based on transition metals $[42,43]$, the solid hydrogen accumulation, storage systems that utilize nanocomposite silicon structures $[44,45]$, and others.

\section{Conflicts of Interest}

The authors declare that there are no conflicts of interest regarding the publication of this paper.

\section{References}

[1] M. C. Beard, J. M. Luther, and A. J. Nozik, "The promise and challenge of nanostructured solar cells," Nature Nanotechnology, vol. 9, no. 12, pp. 951-954, 2014.

[2] Z. I. Alferov, V. M. Andreev, and V. D. Rumyantsev, "Solar photovoltaics: trends and prospects," Semiconductors, vol. 38, no. 8, pp. 899-908, 2004.

[3] B. Yan, G. Yue, X. Xu, J. Yang, and S. Guha, "High efficiency amorphous and nanocrystalline silicon solar cells," Physica Status Solidi, vol. 207, no. 3, pp. 671-677, 2010.

[4] N. S. Lewis, “Toward cost-effective solar energy use," Science, vol. 315, no. 5813, pp. 798-801, 2007.

[5] R. Søndergaard, M. Hösel, D. Angmo, T. T. Larsen-Olsen, and F. C. Krebs, "Roll-to-roll fabrication of polymer solar cells," Materials Today, vol. 15, no. 1-2, pp. 36-49, 2012. 
[6] M. Birkholz, B. Selle, E. Conrad, K. Lips, and W. Fuhs, "Evolution of structure in thin microcrystalline silicon films grown by electron-cyclotron resonance chemical vapor deposition," Journal of Applied Physics, vol. 88, no. 7, pp. 4376-4379, 2000.

[7] B. Rech, T. Roschek, J. Müller, S. Wieder, and H. Wagner, "Amorphous and microcrystalline silicon solar cells prepared at high deposition rates using RF (13.56 MHz) plasma excitation frequencies," Solar Energy Materials \& Solar Cells, vol. 66, no. 1-4, pp. 267-273, 2001.

[8] M. K. van Veen, C. H. M. van der Werf, and R. E. I. Schropp, "Tandem solar cells deposited using hot-wire chemical vapor deposition," Journal of Non-Crystalline Solids, vol. 338-340, no. 1, pp. 655-658, 2004.

[9] Y. Mai, S. Klein, R. Carius et al., "Improvement of open circuit voltage in microcrystalline silicon solar cells using hot wire buffer layers," Journal of Non-Crystalline Solids, vol. 352, no. 9-20, pp. 1859-1862, 2006.

[10] H. Li, R. H. Franken, R. L. Stolk, C. H. M. van der Werf, J. K. Rath, and R. E. I. Schropp, "Controlling the quality of nanocrystalline silicon made by hot-wire chemical vapor deposition by using a reverse $\mathrm{H}_{2}$ profiling technique," Journal of Non-Crystalline Solids, vol. 354, no. 19-25, pp. 2087-2091, 2008.

[11] R. Amrani, F. Pichot, L. Chahed, and Y. Cuminal, "Amorphousnanocrystalline transition in silicon thin films obtained by argon diluted silane PECVD," Crystal Structure Theory and Applications, vol. 1, pp. 57-61, 2012.

[12] G. Fugallo and A. Mattoni, "Thermally induced recrystallization of textured hydrogenated nanocrystalline silicon," Physical Review B: Condensed Matter and Materials Physics, vol. 89, no. 4, Article ID 045301, 10 pages, 2014.

[13] O. Nast and A. J. Hartmann, "Influence of interface and $\mathrm{Al}$ structure on layer exchange during aluminum-induced crystallization of amorphous silicon," Journal of Applied Physics, vol. 88, no. 2, pp. 716-724, 2000.

[14] M. Jeon, C. Jeong, and K. Kamisako, "Tin induced crystallisation of hydrogenated amorphous silicon thin films," Materials Science and Technology, vol. 26, no. 7, pp. 875-878, 2010.

[15] M. A. Mohiddon and M. G. Krishna, "Growth and optical properties of $\mathrm{Sn}-\mathrm{Si}$ nanocomposite thin films," Journal of Materials Science, vol. 47, no. 19, pp. 6972-6978, 2012.

[16] D. Van Gestel, I. Gordon, and J. Poortmans, "Aluminuminduced crystallization for thin-film polycrystalline silicon solar cells: achievements and perspective," Solar Energy Materials \& Solar Cells, vol. 119, pp. 261-270, 2013.

[17] A. Mohiddon and G. Krishna, "Metal induced crystallization," in Crystallization-Science and Technology, A. Marcello, Ed., pp. 461-480, InTech, 2012.

[18] V. V. Voitovych, V. B. Neimash, N. N. Krasko et al., “The effect of Sn impurity on the optical and structural properties of thin silicon films," Semiconductors, vol. 45, no. 10, pp. 1281-1285, 2011.

[19] V. B. Neimash, V. M. Poroshin, A. M. Kabaldin et al., "Microstructure of thin Si-Sn composite films," Ukrainian Journal of Physics, vol. 58, no. 9, pp. 865-871, 2013.

[20] V. Neimash, V. Poroshin, P. Shepeliavyi et al., "Tin induced a-Si crystallization in thin films of Si-Sn alloys," Journal of Applied Physics, vol. 114, no. 21, Article ID 213104, 2013.

[21] V. B. Neimash, A. O. Goushcha, P. E. Shepeliavyi et al., "Mechanism of tin-induced crystallization in amorphous silicon," Ukrainian Journal of Physics, vol. 59, no. 12, pp. 1168-1176, 2014.

[22] V. B. Neimash, A. O. Goushcha, P. Y. Shepeliavyi et al., "Self-sustained cyclic tin induced crystallization of amorphous silicon," Journal of Materials Research, vol. 30, no. 20, pp. 31163124, 2015.

[23] V. Neimash, P. Shepelyavyi, G. Dovbeshko et al., "Nanocrystals growth control during laser annealing of $\mathrm{Sn}:(\alpha-\mathrm{Si})$ composites," Journal of Nanomaterials, vol. 2016, Article ID 7920238, 8 pages, 2016.

[24] H. Richter, Z. P. Wang, and L. Ley, "The one phonon Raman spectrum in microcrystalline silicon," Solid State Communications, vol. 39, no. 5, pp. 625-629, 1981.

[25] I. H. Campbell and P. M. Fauchet, “The effects of microcrystal size and shape on the one phonon Raman spectra of crystalline semiconductors," Solid State Communications, vol. 58, no. 10, pp. 739-741, 1986.

[26] R.-P. Wang, G.-W. Zhou, Y.-L. Liu et al., "Raman spectral study of silicon nanowires: high-order scattering and phonon confinement effects," Physical Review B: Condensed Matter and Materials Physics, vol. 61, no. 24, pp. 16827-16832, 2000.

[27] A. Hiraki, "Low temperature reactions at Si/metal interfaces; What is going on at the interfaces?" Surface Science Reports, vol. 3, no. 7, pp. 357-412, 1983.

[28] S. A. Akhmanov, V. I. Emel'yanov, N. I. Koroteev, and V. N. Seminogov, "Interaction of powerful laser radiation with the surfaces of semiconductors and metals: nonlinear optical effects and nonlinear optical diagnostics," Uspekhi Fizicheskih Nauk, vol. 147 , no. 12 , p. $675,1985$.

[29] R. Burbelo, D. Andrusenko, M. Isaiev, and A. Kuzmich, "Laser photoacoustic diagnostics of advanced materials with different structure and dimensions," Archives of Metallurgy and Materials, vol. 56, no. 4, pp. 1157-1162, 2011.

[30] R. Burbelo, M. Isaiev, and A. Kuzmich, "Evolution of temperature distribution in implanted Si-based structures: Pulse mode of laser irradiation," Ukrainian Journal of Physics, vol. 55, no. 3, pp. 317-321, 2010.

[31] M. Isaiev, V. Kuryliuk, A. Kuzmich, and R. Burbelo, "Photothermal transformation in heterogeneous semiconductors structures under its pulse laser irradiation: Role of electron-hole diffusion," Archives of Metallurgy and Materials, vol. 58, no. 4, pp. 1351-1354, 2013.

[32] G. Grimvall, Ed., Thermophysical Properties of Materials, Elsevier Science, Amsterdam, Netherlands, 1999.

[33] G. K. M. Thutupalli and S. G. Tomlin, "The optical properties of amorphous and crystalline silicon," Journal of Physics C: Solid State Physics, vol. 10, no. 3, pp. 467-477, 1977.

[34] S. Adachi and H. Mori, "Optical properties of fully amorphous silicon," Physical Review B, vol. 62, no. 15, pp. 10158-10164, 2000.

[35] D. R. Queen, The Specific Heat of Pure and Hydrogenated Amorphous Silicon, University of California, Berkeley, Calif, USA, 2011.

[36] B. L. Zink, R. Pietri, and F. Hellman, "Thermal conductivity and specific heat of thin-film amorphous silicon," Physical Review Letters, vol. 96, no. 5, Article ID 055902, 2006.

[37] S. P. Rodichkina, L. A. Osminkina, M. Isaiev et al., "Raman diagnostics of photoinduced heating of silicon nanowires prepared by metal-assisted chemical etching," Applied Physics B: Lasers and Optics, vol. 121, no. 3, pp. 337-344, 2015.

[38] M. Isaiev, O. Didukh, T. Nychyporuk, V. Timoshenko, and V. Lysenko, "Anisotropic heat conduction in silicon nanowire network revealed by Raman scattering," Applied Physics Letters, vol. 110, no. 1, Article ID 011908, 2017. 
[39] O. Plaksin, Y. Takeda, H. Amekura, N. Kishimoto, and S. Plaksin, "Saturation of nonlinear optical absorption of metalnanoparticle composites," Journal of Applied Physics, vol. 103, no. 11, Article ID 114302, 2008.

[40] G. Conibeer, “Third-generation photovoltaics," Materials Today, vol. 10, no. 11, pp. 42-50, 2007.

[41] G. Conibeer, I. Perez-Wurfl, X. Hao, D. Di, and D. Lin, "Si solid-state quantum dot-based materials for tandem solar cells," Nanoscale Research Letters, vol. 7, no. 1, p. 193, 2012.

[42] V. G. Litovchenko, "On some important results in semiconductor surface science obtained in Ukraine during the independence years (1991-2016)," Ukrainian Journal of Physics, vol. 62, no. 1, pp. 80-95, 2017.

[43] V. G. Litovchenko, T. I. Gorbanyuk, V. S. Solntsev, and A. A. Evtukh, "Mechanism of hydrogen, oxygen and humidity sensing by $\mathrm{Cu} / \mathrm{Pd}$-porous silicon-silicon structures," Applied Surface Science, vol. 234, no. 1-4, pp. 262-267, 2004.

[44] A. I. Manilov and V. A. Skryshevsky, "Hydrogen in porous silicon-a review," Materials Science and Engineering: B, vol. 178, no. 15, pp. 942-955, 2013.

[45] V. Lysenko, J. Vitiello, B. Remaki, D. Barbier, and V. Skryshevsky, "Nanoscale morphology dependent hydrogen coverage of meso-porous silicon," Applied Surface Science, vol. 230, no. 14, pp. 425-430, 2004. 


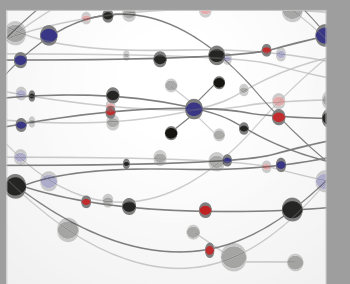

The Scientific World Journal
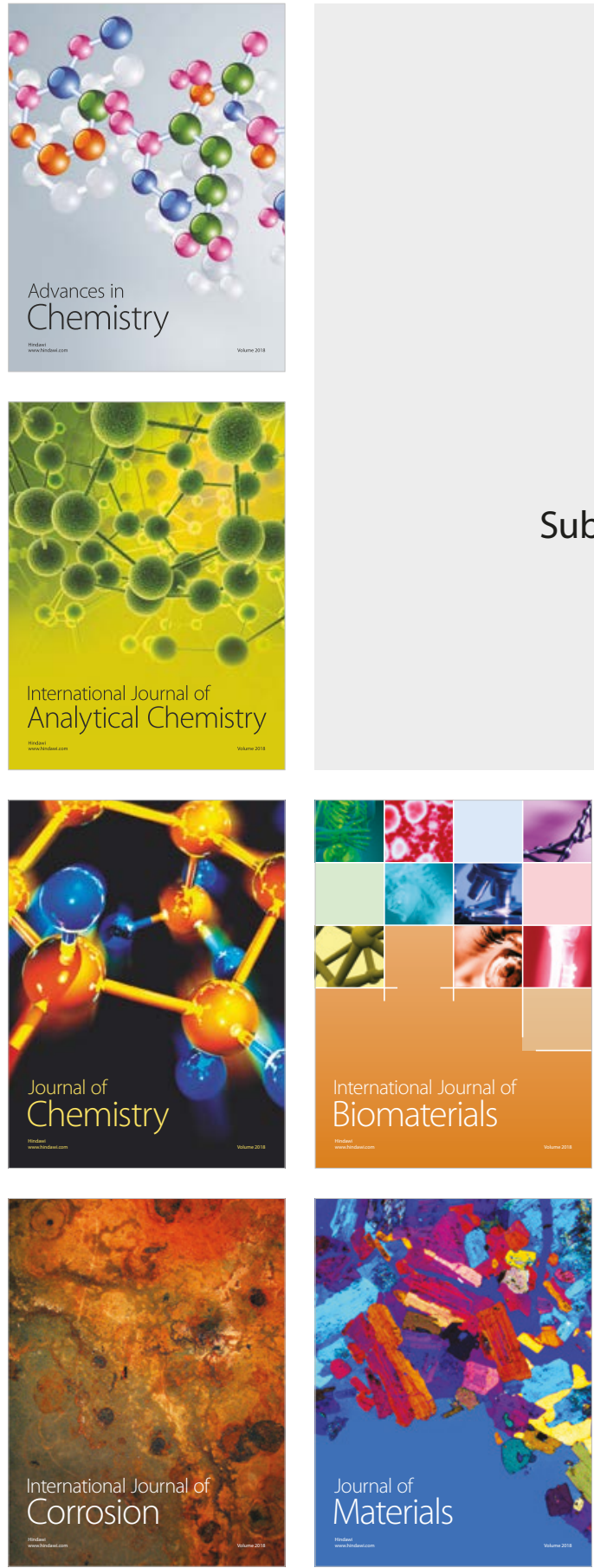

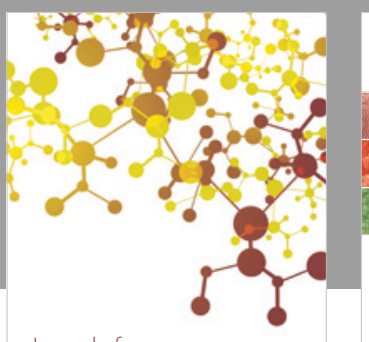

Journal of

Applied Chemistry
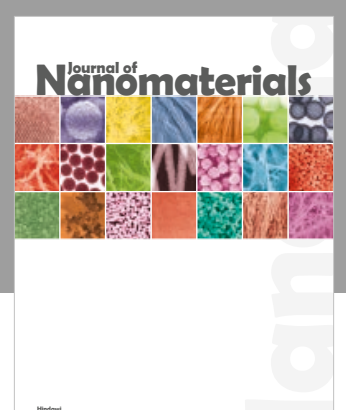

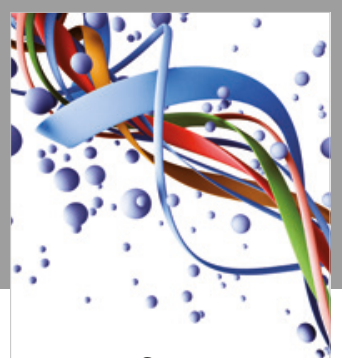

Scientifica

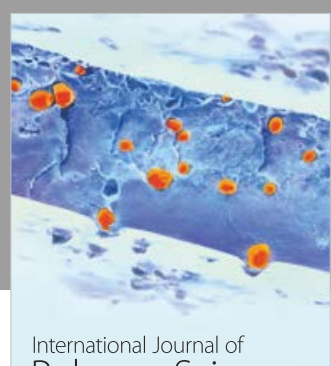

Polymer Science

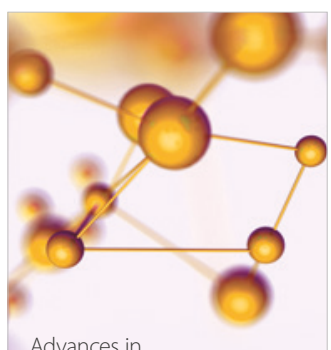

Physical Chemistry
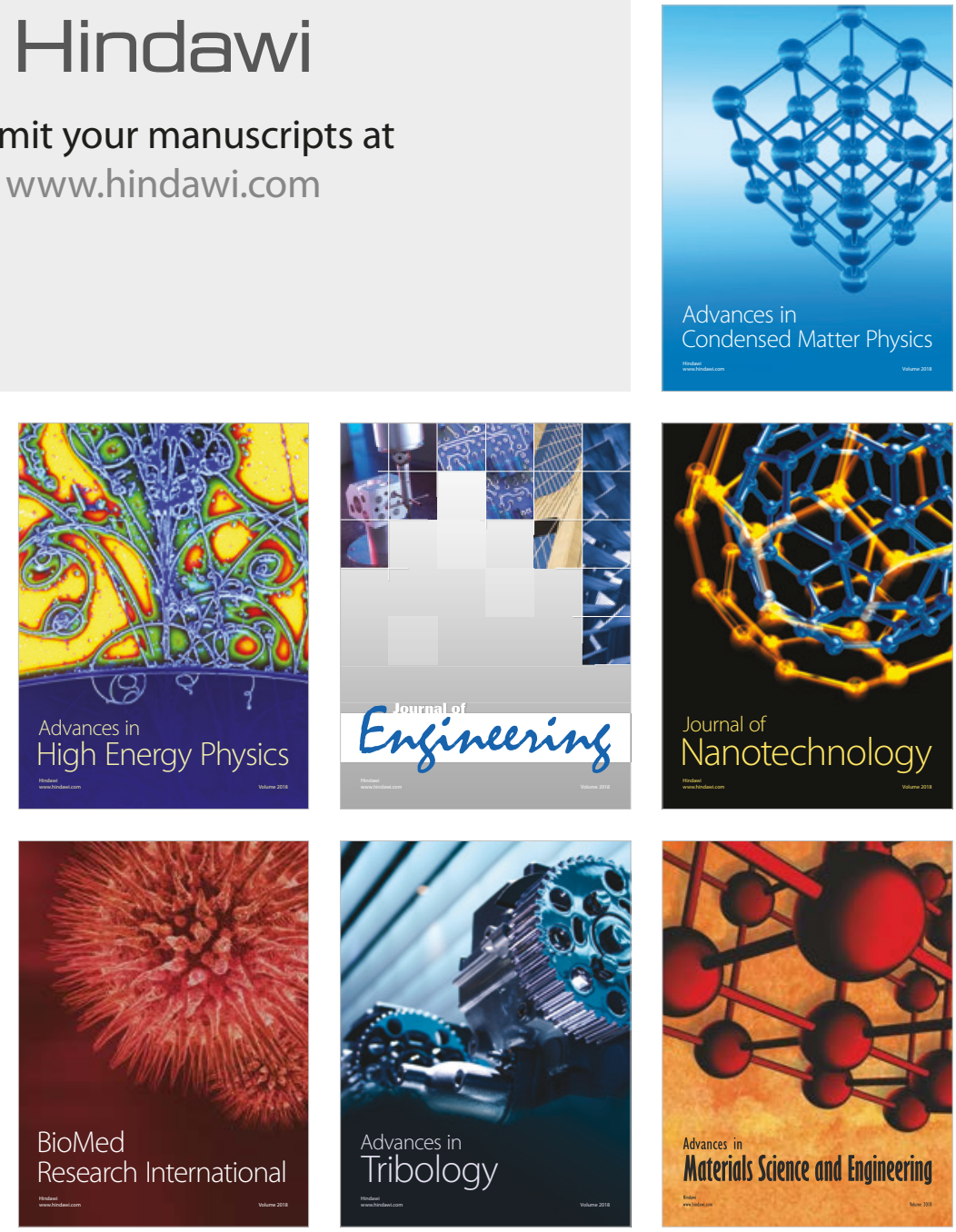